\title{
A GLOSS ON A THEOREM OF FURSTENBERG
}

\author{
LESTER E. DUBINS ${ }^{1}$
}

\begin{abstract}
ABSTRACr. Certain refinements are offered for Furstenberg's ergodic-theoretic version of Szemeredi's theorem.
\end{abstract}

Furstenberg [1977] has proven a significant generalization of a theorem of Poincaré, which, with no real loss, can be formulated thus: If $k$ is a positive integer and $B_{1}, B_{2}, \ldots$ is a stationary sequence of events of positive probability in a countably additive probability space, then there is a $k$-progression, $K$, such that $B_{K}=\cap B_{i}(k \in K)$ has positive probability. (A $k$-progression is a set of $k$ integers of the form $\{a, a+b, a+2 b, \ldots, a+(k-1) b\}$ with $a \geqslant 0, b>0$.)

The present paper observes that neither the hypothesis of countable additivity nor of stationarity is needed. Moreover, the probability of $B_{K}$ can be bounded from below by a $\delta>0$ which depends only on $k$ and $p=P\left(B_{1}\right)$. These facts are immediate corollaries to:

TheOREM 1. Let $p>0$ and let $k$ be $a$ positive integer. Then there is $a \delta>0$ and $a$ positive integer $n$ such that, for every $n$-tuple of events $B_{1}, \ldots, B_{n}$ of average probability at least $p$, there is a k-progression $K \subset\{1, \ldots, n\}$ for which $\cap B_{i}(i \in K)$ has probability at least $\delta$.

This form of Furstenberg's theorem follows by an argument which he chose not to provide in [1977]. Indeed, it is a simple consequence of Szemeredi's theorem [1975] on the existence of arbitrarily long arithmetic sequences in each set of integers of positive density. But it is convenient first to provide a trivial lemma.

Lemma 1. Let $B_{1}, \ldots, B_{n}$ be events of average probability at least $p$ and let $l$ be $a$ positive integer less than $n$. Then there is a subset $X$ of $\{1, \ldots, n\}$ of cardinality $l$ such that

$$
P\left(\cap B_{i}(i \in X)\right) \geqslant\left(p-\frac{l}{n}\right) /\left(\begin{array}{l}
n \\
l
\end{array}\right) .
$$

Proof of Lemma 1. Let $Y$ be the number of $B$ that occur. Since $Y$ is at most $n$ on the event $(Y \geqslant l)$ and is at most $l-1$ on its complement, the following inequality (sharp) is easily obtained.

$$
P(Y \geqslant l) \geqslant\left(\frac{P Y}{n}-\frac{l-1}{n}\right)\left(1-\frac{l-1}{n}\right)^{-1} .
$$

Received by the editors July $28,1982$.

1980 Mathematics Subject Classification. Primary 10L20, 28D05, 60G10.

Key words and phrases. Ergodic, probability.

'Supported by National Science Foundation Grant MCS80-02535.

(C)1983 American Mathematical Society $0002-9939 / 83 \$ 1.00+\$ .25$ per page 
(In (2), the precision (expectation) of $Y$ is designated by $P Y$ as accords with a notational innovation of de Finetti.)

For the purposes of this note, this weaker inequality suffices:

$$
P(Y \geqslant l) \geqslant \frac{P Y}{n}-\frac{l}{n} \text {. }
$$

Plainly, the event $Y \geqslant l$ is the union of the events $\cap B_{i}(i \in X)$ as $X$ ranges over $[n]^{l}$, the subsets of $\{1, \ldots, n\}$ of cardinality $l$. Therefore,

$$
P(Y \geqslant l) \leqslant \sum P\left(\cap B_{i}(i \in X)\right) \leqslant\left(\begin{array}{c}
n \\
l
\end{array}\right) \max P\left(\bigcap B_{i}(i \in X)\right),
$$

as $X$ ranges over $[n]^{l}$. So, for some $X \in[n]^{\prime}$,

$$
\begin{aligned}
P\left(\cap B_{i}(i \in X)\right) & \geqslant P(Y \geqslant l) /\left(\begin{array}{l}
n \\
l
\end{array}\right) \geqslant\left(\frac{P Y}{n}-\frac{l}{n}\right) /\left(\begin{array}{c}
n \\
l
\end{array}\right) \\
& \geqslant\left(p-\frac{l}{n}\right) /\left(\begin{array}{l}
n \\
l
\end{array}\right),
\end{aligned}
$$

where the second inequality obtains in view of (3), and the third by hypothesis.

Let $\gamma_{k}(n)$ be the least integer $l$ such that, if $X$ is a subset of $\{1, \ldots, n\}$ of cardinality $l$, then $X$ includes a $k$-progression. Szemeredi [1975] has shown that $\gamma_{k}(n) / n$ converges to 0 as $n \rightarrow \infty$.

Proof of Theorem 1. By Szemeredi's theorem, there is an $n=n(p, k)$ such that $\gamma_{k}(n)<n p / 2$. For $l=\gamma_{k}(n)$, let $\delta$ be $p / 2\left(\begin{array}{l}n \\ l\end{array}\right)$. That $(\delta, n)$ satisfies Theorem 1 can be verified, thus. Let $B_{1}, \ldots, B_{n}$ be events of average possibility at least $p$. By Lemma 1 , there is an $X \subset\{1, \ldots, n\}$ of cardinality $l$ such that (1) holds. Since $l / n<p / 2$, the right-hand side of (1) is at least $\delta$. So $\cap B_{i}(i \in X)$ has probability no less than $\delta$. Since $X$ is of cardinality $\gamma_{k}(n), X$ includes a $k$-progression, $K$. Plainly, $\cap B_{i}(i \in K)$ includes $\cap B_{i}(i \in X)$. So it, too, has probability no less than $\delta$.

\section{REFERENCES}

[1977] Harry Furstenberg, Ergodic behavior of diagonal measures and a theorem of Szemeredi on arithmetic progressions, J. Analyse Math. 36, 204-256.

[1975] E. Szemeredi, On sets of integers containing no $k$ elements in arithmetic progression, Acta Arith. 27, 199-245.

Department of Mathematics, University of California, Berkeley, California 94720 